\title{
La gestione della linea infusionale venosa
}

\author{
A. Nurcis
}

Responsabile Commerciale Area Critica, Gadomed, Genova

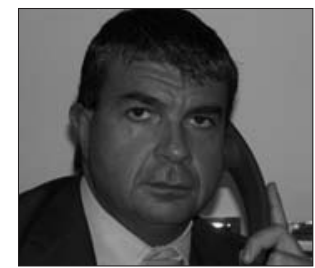

I dispositivi intravascolari sono diventati d'uso comune nelle pratiche medico-assistenziali in quanto, dando accesso al circolo ematico, creano il tramite per la somministrazione della terapia endovenosa (IV). Da ciò si evince la fondamentale importanza per il personale medico e infermieristico di conoscere, valutare e gestire i vari tipi di dispositivi intravascolari al fine di una corretta somministrazione e gestione della IV.

Il cateterismo venoso può essere così suddiviso.

Esistono alcuni fattori discriminanti per la scelta di un Catetere Venoso Centrale $(\mathrm{CVC})$ :

Tempo: infusioni di breve, media o lunga durata.

Monitoraggio: Pressione Venosa Centrale.

Tipologia delle soluzioni da infondere: chemioterapici, antibiotici, emoderivati, soluzioni ipertoniche, NPT(nutrizione parenterale totale) e, in generale, qualsiasi tipo di soluzione ad elevata osmolarità.

Necessità d'infusioni rapide .

La durata infusionale e il tipo di soluzione da infondere sono le variabili che determinano il tipo di materiale da impiegare.

I materiali comunemente utilizzati sono:

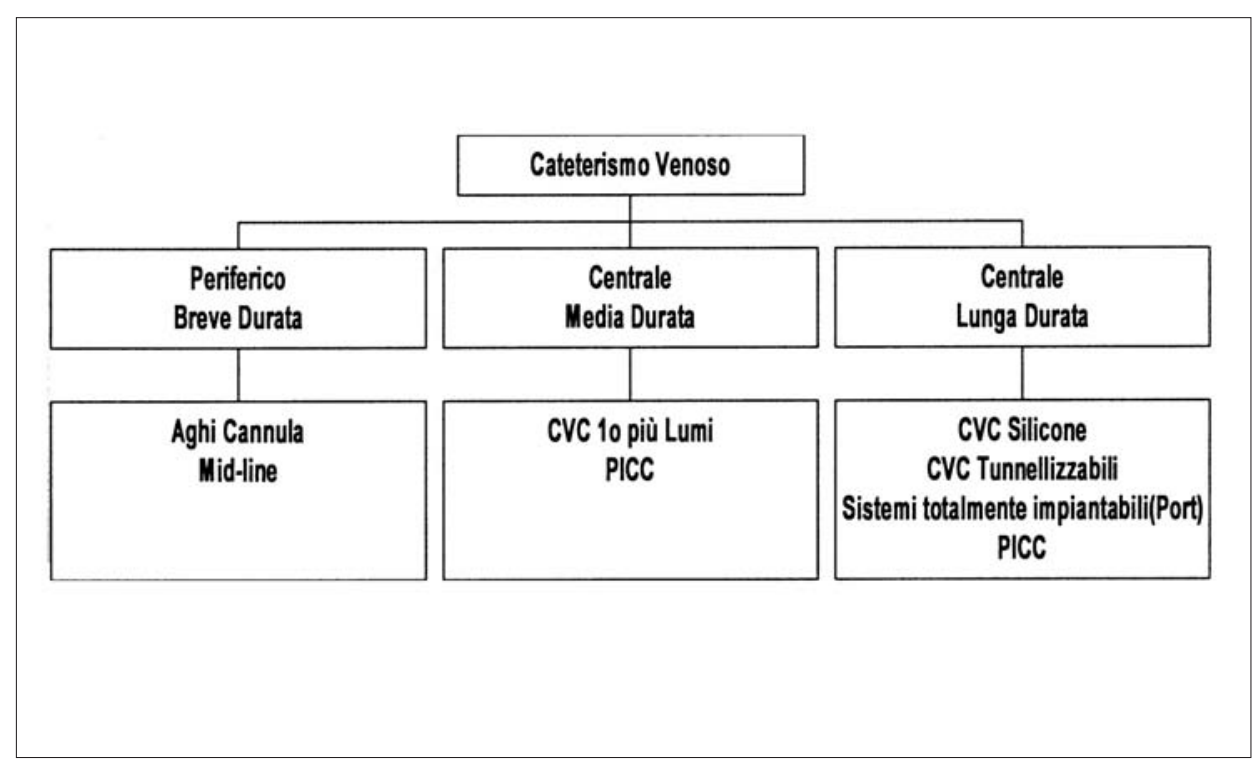

- PVC (Polivinilcloruro): materia plastica alla quale possono essere aggiunti numerosi additivi (per esempio: ftalati, coloranti, opacizzanti, lubrificanti ecc.) volti a migliorarne le caratteristiche di base e a ottimizzarne l'utilizzo.

Il contatto con le soluzioni infuse ne provoca però il rilascio nel flusso ematico.

\section{Vantaggi}

- Morbidezza

- Varietà

- Basso costo

\section{$\underline{\text { Svantaggi }}$}

- Rilascio di additivi

- Cattiva compatibilità farmacologia

- Inadatto ad alcune applicazioni (no pompa siringa, no monitoraggio arterioso) 
- PUR (PoliUretano): materiale plastico derivante dall'addizione di un poliisocianato e un polipolio.

Essendo questi in grande numero, sono moltissimi i poliuretani ottenibili; tra questi quelli aromatici e alifatici sono i più usati nel cateterismo venoso.

Vantaggi
- Purezza
- Varietà
- Buona biocompatibilità
- Bassa trombogenicità
- Morbidezza
- Elasticità
- Termoplasticità

- PPE (PoliEtilene): poliolefina che presenta caratteristiche ottimali per le infusioni farmacologiche. La sua lavorazione non prevede uso di additivi.

\begin{tabular}{|ll|}
\hline Vantaggi & Svantaggi \\
- Purezza & • Rigidità \\
- Ottima compatibilità farmacologica & • Sensibilità ai raggi UV \\
- Possibile lavorazione su piccoli calibri & \\
- Adatto all'uso in pompa siringa e per & \\
- il monitoraggio arterioso & \\
- Costo relativamente basso & \\
\hline
\end{tabular}

Oltre ai cateteri venosi esistono una serie di accessori studiati sulla base delle necessità terapeutiche.

Gli accessori più comunemente utilizzati nelle terapie in vena sono: rubinetti, rampe, tappi, prolunghe e raccordi. Questi presidi, assolutamente

\section{Manipolazioni}

Sono tutte quelle procedure esercitate sulla linea infusionale quali:

- Montaggio della linea

- Cambi della linea

- Prelievi/Boli.

- Apertura di un rubinetto

- Eparinizzazione

- Medicazione

Qualsiasi manipolazione porta a contatto il personale con la linea infusionale

\section{Questo contatto comporta dei rischi!}

\section{$\underline{\text { Svantaggi }}$}

- Costo relativamente elevato

- Cattiva compatibilità con i farmaci nitroderivati e calcioantagonisti (Venitrin, ....)

- Inadatto ad alcune applicazioni (no pompa siringa, no monitoraggio arterioso)

indispensabili, rappresentano un potenziale rischio di contaminazione per il paziente, essendo soggetti a plurime manipolazioni da parte degli operatori. Esaminiamo nel dettaglio cosa si intende per manipolazione e quali sono i rischi dovuti alle manipolazioni.

\section{$\underline{\text { Rischi connessi }}$}

- Aumento della carica batterica

- Contaminazione del CVC

- Contaminazioni crociate

- Punture accidentali dell'operatore
Vista la vastità di materiali e i rischi legati alle manipolazioni risulta importante la preparazione di procedure che regolino l'approcio alla linea infusionale; l'insieme di queste procedure si chiama "protocollo".

Nella stesura di un protocollo devo tener presente:

- Identificazione dell'obiettivo generale.

- Identificazione delle figure che si occupano della procedura.

- Identificazione della procedura particolare.

- Identificazione di tutti gli altri obiettivi che compongono una azione $e$ una prestazione.

- Possibilità di dare ad ogni atto un tempo e un costo.

- Possibilità di compiere verifiche ad ogni atto.

- Possibilità di fare cambiamenti ad ogni atto.

Qual è quindi l'obiettivo di una corretta gestione infusionale?

\section{Obiettivo}

- Salvaguardia del malato (riducendo i rischi di contaminazione e ottimizzando le somministrazioni)

- Sicurezza del personale (riducendo il contatto con aghi e flusso ematico)

- Migliorare la qualità del lavoro svolto

- Diminuire il carico di lavoro 
Per raggiungere l'obiettivo risulterà quindi importante l'assemblaggio di materiali idonei e sicuri, seguendo uno schema che terrà conto di tutte le pro- cedure che dovrò effettuare.

Di seguito alcuni esempi di impiego dei

CVC più comunemente utilizzati.

\section{CVC Monolume \\ Punto operativo (boli e prelievi).}

Prolunga (per allontanare le manovre da un luogo sporco).

Rubinetto a tre vie

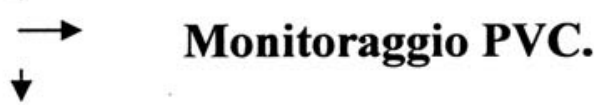

Rampa di rubinetti (gestione farmacologica)
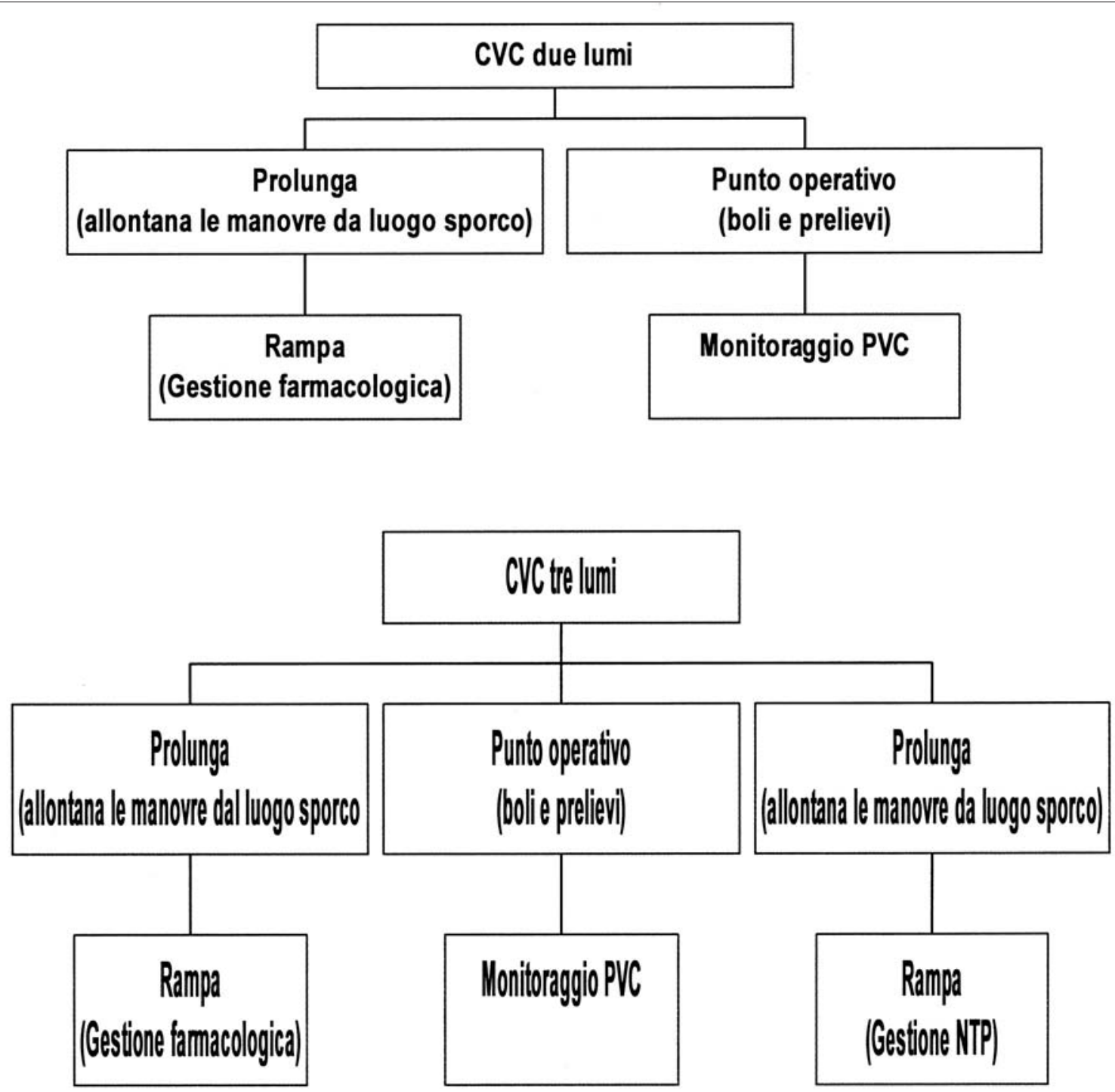


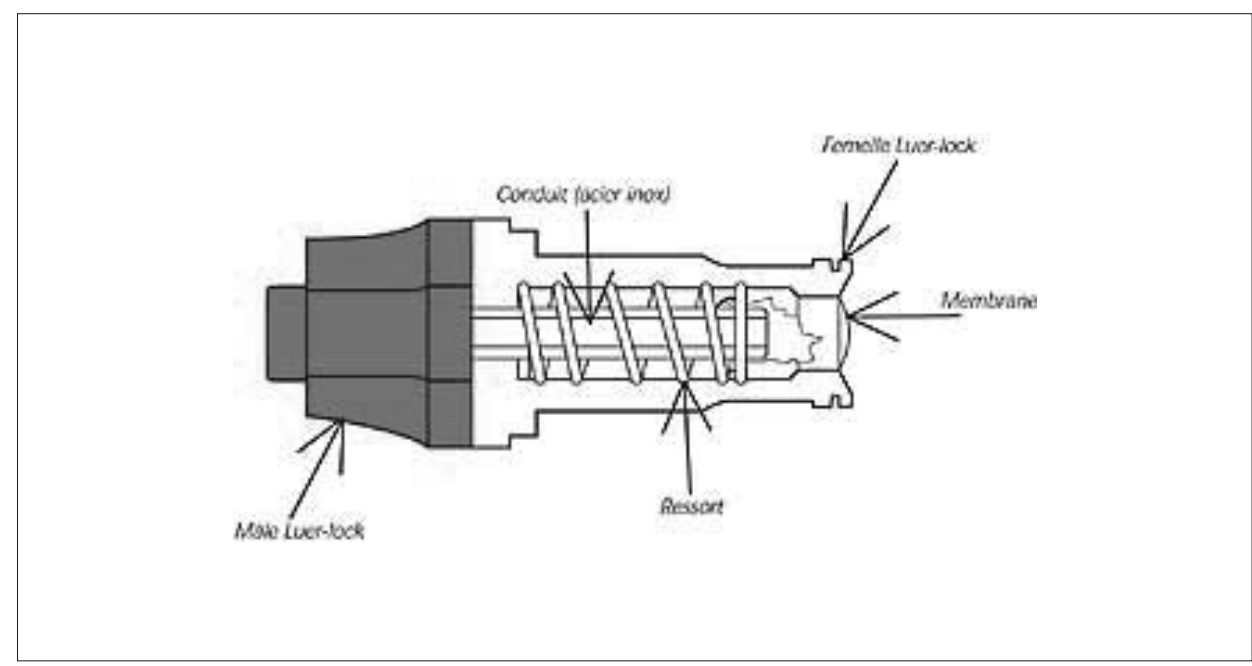

Fig. 1

Per la riduzione dei rischi legati alla gestione della linea infusionale, alcune aziende hanno ideato dei sistemi chiamati needleless (Fig. 1). Questi sistemi hanno molteplici funzioni:

essere usati per effettuare boli o prelievi senza l'ausilio di aghi (evita il rischio di punture accidentali) (Fig. 2).

Essere utilizzati anche all'interno della linea infusionale (tra CVC e prolunga, tra rubinetto e prolunga, tra pompa e prolunga) senza alterare il flusso e garantendo una chiusura ermetica al momento della deconnessione.

Particolarmente importante nella valutazione di questi sistemi risulta la struttura interna che deve consentire un flusso lineare per garantire una buona lettura dell'onda pressoria ed evitare le turbolenze (spesso causa di punti di ristagno).

Risulta inoltre fondamentale verificare la loro ermeticità, che deve garantire una completa ed efficace disinfezione prima di ogni approccio.

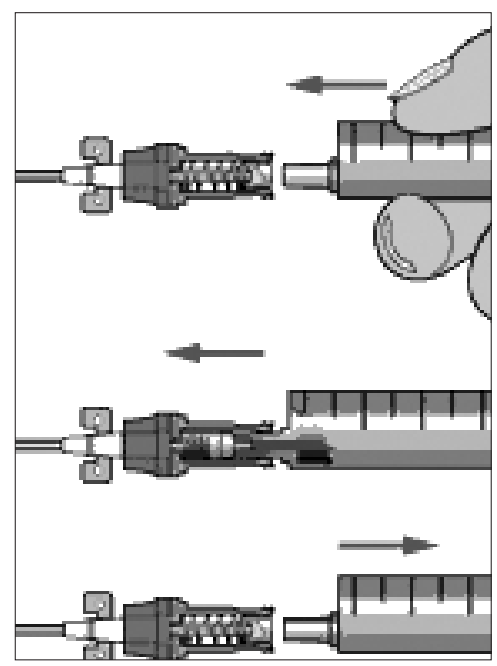

Fig. 2 